\title{
Functional Connectivity in Parkinson's Disease Patients with Mild Cognitive Impairment
}

\author{
Qingguang Wang' \\ Wei $\mathrm{He}^{2}$ \\ Dinghua Liu' \\ Bojun Han' \\ Qitao Jiang ${ }^{3}$ \\ Jiali Niu (10 ${ }^{4}$ \\ Yunlong Ding ${ }^{5}$
}

'Department of Neurology, The Affiliated Jiangyin People's Hospital of Southeast University Medical College, Jiangyin, People's Republic of China; ${ }^{2}$ Department of Physical Medicine and Rehabilitation, The Affiliated Jiangyin People's Hospital of Southeast University Medical College, Jiangyin, People's Republic of China; ${ }^{3}$ Department of Medical Imaging, The Affiliated Jiangyin People's Hospital of Southeast University Medical College, Jiangyin, People's Republic of China; ${ }^{4}$ Department of Clinical Pharmacy, Jingjiang People's Hospital, Jingjiang, People's Republic of China; ${ }^{5}$ Department of Neurology, Jingjiang People's Hospital, jingjiang, People's Republic of China
Correspondence: Jiali Niu

Department of Clinical Pharmacy, Jingjiang People's Hospital, No. 28, Zhongzhou

Road, Jingjiang, 2 I4500, Jiangsu, People's

Republic of China

Tel +86-15I89921635

Email jialiniu 123@sina.com

Yunlong Ding

Department of Neurology, Jingjiang

People's Hospital, No. 28, Zhongzhou

Road, Jingjiang, 2 | 4500, Jiangsu, People's

Republic of China

Tel +86-I5861035745

Email dingyunlong66@sina.com
Objective: To explore the alteration of pattens of anatomical and functional connectivity (FC) of posterior cingulate cortex (PCC) in Parkinson's disease (PD) patients with cognitive dysfunction and the relationship between the connection strengths and cognitive state.

Methods: We prospectively enrolled 20 PD patients with mild cognitive impairment (PD$\mathrm{MCI}$ ), 13 PD patients with normal cognition (PD-NC) and 13 healthy controls (HCs). By collecting, preprocessing and $\mathrm{FC}$ analyzing resting-state functional magnetic resonance imaging (rs-fMRI) data, we extracted default mode network (DMN) patterns, compared the differences in DMN between the three groups and the analyzed the correlation between FC value with the commonly used neuropsychological testing.

Results: The PD-MCI showed significant worse performances in general cognition, and PD$\mathrm{NC}$ and $\mathrm{HCs}$ showed comparable performances of cognitive function. Cognitive-related differences in DMN were detected in the bilateral precuneus (BPcu). Compared with the HCs, PD-NC and PD-MCI showed significantly decreased FC within BPcu (both P $<0.001$ ). For PD-MCI, the rho of the Fisher's Z-transformed FC ( $\mathrm{zFC}$ ) value within BPcu with the TMTA, DSST and CFT-20min were 0.50, 0.66 and 0.47, respectively. For PD-NC, the rho of the $\mathrm{zFC}$ value within BPcu with the MMSE was 0.58 .

Discussion: BPcu was the cognitive-related region in DMN. As cognition declines, FC within BPcu weakens. For PD-MCI, the higher the FC values within BPcu were likely to be related to the better the performances of TMTA, DSST and CFT-20 min DR, which needs to be further confirmed by large-sample studies.

Keywords: Parkinson's disease, mild cognitive impairment, resting-state functional magnetic resonance imaging, functional connectivity

\section{Introduction}

Parkinson's disease (PD) is a chronic and progressive neurodegenerative disorder, decreasing the quality of life and placing serious social and economic burdens. ${ }^{1}$ In China, the prevalence of PD is 1700 per 100,000 in individuals older than 65 years, which is similar to the rate reported in developed countries. ${ }^{2,3} \mathrm{PD}$ is usually characterized by motor symptoms that the main clinical manifestations are bradykinesia, rest tremor present, muscular rigidity and postural instability ${ }^{4}$ but may lead to mild cognitive impairment and dementia. Evidence implicates that patients with PD are six times more likely to develop dementia than healthy people. ${ }^{5}$ PD with mild cognitive impairment (PD-MCI) has attracted much attention in recent years.

The pathogenesis and pathogenesis of PD-MCI are not yet fully understood. In recent years, researchers have conducted in-depth studies on the pathogenesis and clinical features of PD-MCI. Among them, the research progress of imaging, 
especially magnetic resonance imaging (MRI) has shown potential significance for early screening and early diagnosis of PD-MCI.

MRI is one of the most widely used methods studying PD-MCI in recent years. Studies have shown that MRI technology provides a new evidence for early detection of PD-MCI. Current imaging techniques mainly include anatomical structure MRI imaging region-of-interest (ROI), voxel-based morphometry (VBM), cortical thickness analysis, white matter imaging technology, magnetic resonance diffusion tensor imaging (DTI), functional magnetic resonance imaging (fMRI) and perfusion imaging technology. At present, there are few reports on the application of resting state fMRI (rs-fMRI) technology to study PD-MCI.

In order to investigate the cognitive-related changes of gray matter structure and function in PD patients, this study using rs-fMRI to assess the pattens of anatomical and functional connectivity (FC) damage in $\mathrm{PD}$ patients with normal cognition (PD-NC) and PD-MCI. Furthermore, we analyze the relationship between the connection strengths and cognitive state.

\section{Materials and Methods \\ Participants}

Twenty PD-MCI, 13 PD-NC and 13 healthy controls (HCs) were employed in the present study through newspaper advertisements. All subjects underwent clinical evaluations and MRI scans. One PD-MCI and one PD-NC were excluded from the imaging analysis because of the excessive motion artifacts. The study was approved by the Research Ethics Committee of the Affiliated Jiangyin People's Hospital of Southeast University Medical College and written informed consent was obtained from all participants.

\section{Clinical Evaluation}

Each subject underwent comprehensive clinical evaluations, including the demographic information, history of past illness and neuropsychological testing. Mini-Mental State Examination (MMSE) was used to assess the general cognition. The neuropsychological battery mainly comprised of Auditory Verbal Learning Test-20-min delayed recall (AVLT-20min DR), the Rey-Osterrieth Complex Figure Test (CFT-20min DR), Trail Making Test (TMT)A and B, Verbal Fluency Test (VFT), Digital Symbol Substitution Test (DSST) and Clock Drawing Test (CDT) were used to evaluate the episodic memory, visuospatial function, information processing speed and executive function.

\section{Inclusion and Exclusion Criteria}

The diagnosis of PD was made following the 2016 Chinese diagnostic criteria for PD. ${ }^{6}$

The diagnosis of MCI was made following the recommendations of Petersen et $\mathrm{al}^{7}$ and others: ${ }^{8,9}$ (a) subjective memory impairment corroborated by the subject and an informant; (b) objective memory performance documented by an AVLT-20min DR score less than or equal to $1.5 \mathrm{SD}$ of age- and education-adjusted norms (cut-off of $\leq 4$ correct responses on 12 items for patients with $\geq 8$ years of education); (c) MMSE score of 24 or lower; (d) Clinical Dementia Rating (CDR) of 0.5; (e) no or minimal impairment in activities of daily living; (f) absence of dementia or insufficient dementia to meet the National Institute of Neurological and Communicative Disorders and Stroke and the Alzheimer's Disease and Related Disorders Association (NINCDS-ADRDA) Alzheimer's criteria. In addition, the controls were required to have a CDR of 0 , an MMSE score $\geq 26$, and an AVLT-20min DR score $\geq 4$ for subjects with 8 or more years of education.

Participants were excluded from the study if they had a history of dementia and mental illness, severe heart, liver, and kidney dysfunction, malignant tumors and endocrine and metabolic diseases, left-handed, cerebrovascular disease, trauma, progressive ascending paralysis confirmed, multiple system atrophy, and cortical basal ganglia disease.

\section{MRI Data Acquisition}

The subjects were scanned using a General Electric 1.5 Tesla scanner (General Electric Medical Systems, USA) with a homogeneous birdcage head coil (16 channels). Subjects lay supine with the head snugly fixed by a belt and foam pads to minimize head motion. Conventional axial fast relaxation fast spin echo sequence T2 weighted anatomic MR images were obtained to rule out major white matter changes, cerebral infarction or other lesions: repetition time $(\mathrm{TR})=3500 \mathrm{~ms}$; echo time $(\mathrm{TE})=103$ $\mathrm{ms}$; flip angle $(\mathrm{FA})=90^{\circ}$; acquisition matrix $=320 \times 192$; field of view $(\mathrm{FOV})=240 \times 240 \mathrm{~mm}$; thickness $=6.0 \mathrm{~mm}$; gap $=0 \mathrm{~mm}$; no. of excitations $(\mathrm{NEX})=2.0$. Highresolution T1-weighted axial images covering the whole brain were acquired using a 3D spoiled gradient echo sequence as follows: $\mathrm{TR}=9.9 \mathrm{~ms} ; \mathrm{TE}=2.1 \mathrm{~ms} ; \mathrm{FA}=$ 
$15^{\circ} ;$ acquisition matrix $=256 \times 192 ; \mathrm{FOV}=$ $240 \mathrm{~mm} \times 240 \mathrm{~mm}^{2}$; thickness $=2.0 \mathrm{~mm}$; gap $=0 \mathrm{~mm}$. The functional scans ( $\mathrm{T} 2 *$ weighted images) involved the acquisition of 30 contiguous axial slices using a GRE-EPI pulse sequence: $\mathrm{TR}=3,000 \mathrm{~ms}$; $\mathrm{TE}=40 \mathrm{~ms} ; \mathrm{FA}=90^{\circ}$; acquisition matrix $=64 \times 64 ; \mathrm{FOV}=240 \times 240 \mathrm{~mm}$; thickness $=4.0 \mathrm{~mm}$; gap $=0 \mathrm{~mm}$ and $3.75 \times 3.75 \mathrm{~mm}^{2}$ inplane resolution parallel to the anterior commissure-posterior commissure line. This acquisition sequence generated 142 volumes in $7 \mathrm{~min}$ and $6 \mathrm{~s}$. All subjects have eyes closed during scanning.

\section{MRI Data Preprocessing}

The preprocessing was performed by using Data Processing Assistant for Resting-State fMRI (DPARSF) (Yan \& Zang, 2010, http://www.restfmri.net), which is based on Statistical Parametric Mapping (SPM5) (http:// www.fil.ion.ucl.ac.uk/spm) and Resting-State fMRI Data Analysis Toolkit (REST, Song et al, 2011. http://www.rest fmri.net). Data analyses of groups were conducted with SPM5 toolkit (http://www.fil.ion.ucl.ac.uk/spm). The first eight volumes of the scanning session were discarded to allow for $\mathrm{T} 1$ equilibration effects. The remaining images were corrected for timing differences and motion effects. No translation or rotation parameters of head motion in any given data set exceeded $\pm 3 \mathrm{~mm}$ or \pm 3 . The resulting images were spatially normalised into the SPM5 Montreal Neurological Institute echo-planar imaging template using the default settings and resampling to $3 \times 3 \times 3 \mathrm{~mm} 3$ voxels, and smoothed with a Gaussian kernel of $6 \times$ $6 \times 6 \mathrm{~mm}$. Then, the linear trend of time courses was removed and the resulting fMRI data were band-pass filtered $(0.01<\mathrm{f}<0.08 \mathrm{~Hz})$.

\section{FC Analysis}

The posterior cingulate cortex (PCC) hub [coordinate in the MNI space: $-2,-45,34]$ was selected to generate a $6-\mathrm{mm}$ radius spherical seed region, ${ }^{10,11}$ followed by coregistration to the functional data. Individual time courses were extracted based on the coregistered seed region. Furthermore, voxel-wise cross-correlation (CC) values between the seed region and the whole brain were calculated. A Fisher's Z-transformation was then applied to improve the normality of the $\mathrm{CC}$ values. Finally, the individual default mode network (DMN) patterns were obtained. Additionally, the mean time series of global, white matter, cerebrospinal fluid signals and head motion parameters were introduced as covariates of no interest.

\section{Voxelwise-Based Grey Matter Volume Correction}

To explore the FC changes that cannot be attributed to anatomical difference, the voxel's likelihood of containing grey matter was introduced as nuisance variables. Firstly, VBM was used to explore grey matter volume maps of every subject. These maps were transformed into the same standard space as the rs-fMRI images using affine linear registration. Further, the resampled data were smoothed as the corresponding functional data $(8 \mathrm{~mm})$. Finally, the resulting voxelwise grey matter volume maps were input as covariates in the analysis of functional data. The voxelwise-based grey matter volume correction was used for each subject. ${ }^{12}$

\section{Statistical Analysis \\ Demographic and Neuropsychological Data}

One-way analysis of variance (ANOVA) was used for normally distributed data. Kruskal Wallis tests followed by Dunn-Bonferroni post hoc were performed as the neuropsychological data were not normally distributed ( $\mathrm{P}<$ 0.05 for Kolmogorov-Smirnov test). The $\chi^{2}$ tests were performed for gender. The SPSS 22.0 software was used and the statistical significance was set at $\mathrm{P}<0.05$.

\section{Group-Level Analyses of the DMN}

Within groups: to determine the patterns of DMN in each of three groups, the spatial maps of DMN IC in each group were submitted to a random-effect analysis using onesample $t$-tests. The thresholds were set at a corrected $\mathrm{P}<$ 0.001, determined by Monte Carlo simulation for multiple comparison (single voxel $\mathrm{P}$ value $=0.001$, a minimum cluster size of $6156 \mathrm{~mm} 3, \mathrm{FWHM}=6 \mathrm{~mm}$; http://afni. nimh.nih.gov/pub/dist/doc/manual/AlphaSim.pdf).

Between groups: ANOVA was performed to explore the different DMN patterns among HCs, PD-NC and PD-MCI. The effects of age, gender and education were corrected. ${ }^{13,14}$ The statistical thresholds were set at an AlphaSim-corrected $\mathrm{P}<0.001$ as determined by Monte Carlo simulation (single voxel $P$ value $=0.001$, a minimum cluster size of $6156 \mathrm{~mm} 3$, FWHM = $6 \mathrm{~mm}$; http://afni.nimh.nih.gov/pub/dist/doc/man ual/AlphaSim.pdf). The regions survived the Monte Carlo simulations were taken as ROIs, and the Fisher's Z-transformed FC (zFC) strengths for each subject were extracted for further analysis.

Characteristic of Disease-Related Difference in DMN Post hoc tests were performed to explore the different DMN patterns of regions with disease-related changes 
Table I Demographic and Neuropsychological Data for HCs, PD with Normal Cognition and PD with $\mathrm{MCl}$

\begin{tabular}{|c|c|c|c|c|}
\hline & PD with $\mathrm{MCl}(\mathrm{n}=20)$ & PD with Normal Cognition $(n=13)$ & $H C(n=13)$ & $\mathbf{P}(\mathbf{K W})$ \\
\hline Age (years) & $72.6 \pm 6.1$ & $70.4 \pm 6.4$ & $70.2 \pm 7.1$ & 0.233 \\
\hline Gender (M/F) & $12 / 8$ & $7 / 6$ & $4 / 9$ & 0.247 \\
\hline Education (years) & $11.7 \pm 3.3$ & $12.2 \pm 3.9$ & $12.1 \pm 3.5$ & 0.688 \\
\hline MMSE & $26.9 \pm 3.1 * * *$ & $28.5 \pm 1.5$ & $28.8 \pm 1.4$ & $<0.001$ \\
\hline AVLT-20min DR & $2.9 \pm 1.2^{* * *}$ & $6.9 \pm 1.1$ & $7.7 \pm 1.5$ & $<0.001$ \\
\hline CFT-20min DR & $13.3 \pm 4.9 * * *$ & $17.5 \pm 4.5$ & $19.2 \pm 4.9$ & $<0.001$ \\
\hline CDT & $8.0 \pm 2.1^{*}$ & $9.2 \pm 1.4$ & $8.9 \pm 1.0$ & 0.037 \\
\hline DSST & $33.8 \pm 7.8^{* * *}$ & $41.2 \pm 8.8$ & $40.3 \pm 10.7$ & $<0.001$ \\
\hline TMT-A (second) & $75.8 \pm 14.5^{*}$ & $63.6 \pm 19.8$ & $66.2 \pm 20.1$ & 0.019 \\
\hline VFT & $\mid 7 . I \pm 4.1 * * *$ & $19.9 \pm 4.4$ & $21.1 \pm 5.2$ & $<0.001$ \\
\hline TMT-B (second) & $210.9 \pm 70.1 * * *$ & $197.1 \pm 72.1$ & $|87.3 \pm 74|$. & $<0.001$ \\
\hline
\end{tabular}

Notes: Data was represented as mean \pm SD. Kruskal Wallis tests followed by Dunn-Bonferroni post hoc were performed as the neuropsychological data were not normally distributed $\left(P<0.05\right.$ for Kolmogorov-Smirnov test). The $\chi^{2}$ tests were performed for gender. *Indicates a statistical difference compared with $\mathrm{HC}$. *Indicate $\mathrm{P}<0.05$ and $* * *$ Indicate $\mathrm{P}<0.00 \mathrm{I}$.

Abbreviations: MMSE, Mini-Mental State examination; AVLT-20, Auditory Verbal Learning Test-20-minute delayed recall; CFT20, Rey-Osterrieth Complex Figure Test-20minute delayed recall; CDT, Clock Drawing Test; DSST, Digital Symbol Substitution Test; TMT-A, Trail Making Test-A; VFT, Verbal Fluency Test; TMT-B, Trail Making Test-B.

among three groups. ANOVA analyses followed by the least-significant difference (LSD) analyses were utilized. To explore the cognitive significances of the diseaserelated changes in DMN, the Spearman correlation analyses were performed between the $\mathrm{zFC}$ strengths and the neuropsychological performances. The SPSS 22.0 software was used and the statistical significance was set at $\mathrm{P}<0.05$.

\section{Results}

\section{Demographic and Cognitive Data}

As shown in Table 1, the PD-MCI showed significant worse performances in general cognition (ie, MMSE), episodic memory (ie, AVLT-20min DR and CFT-20min DR), visuospatial function (ie, CDT), information processing speed (ie, DSST and TMT-A) and executive function (ie, TMT-B and VFT, all P < 0.05). Furthermore, the PD$\mathrm{NC}$ and HCs showed comparable performances of cognitive function (all $\mathrm{P}>0.05$ ). It should be noted that there were no significant differences with regard to demographic data among the three groups (all $\mathrm{P}>0.05$ ).

\section{Identification of Disease-Related Differences in DMN}

As illustrated by the one-sample $t$-test, the DMN patterns for each group were obtained by using PCC seed-based FC analysis (shown in Figure 1).

As shown by one-way ANOVA, the disease-related differences in DMN were detected in the bilateral precuneus (BPcu) (shown in Table 2 and Figure 2). It should be noted that the region mentioned above was taken as ROI.

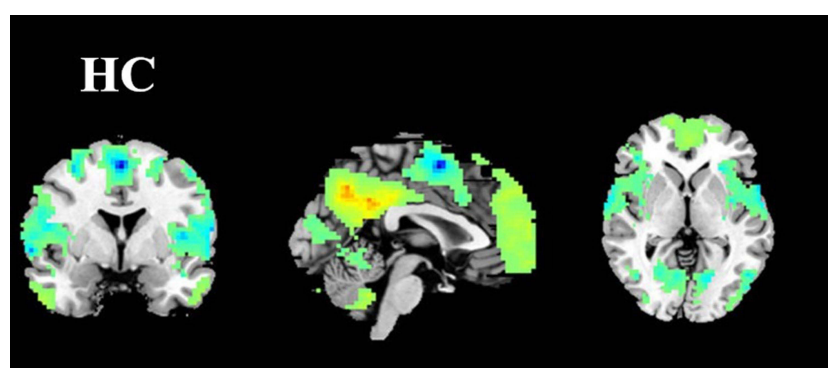

PD with normal cognition

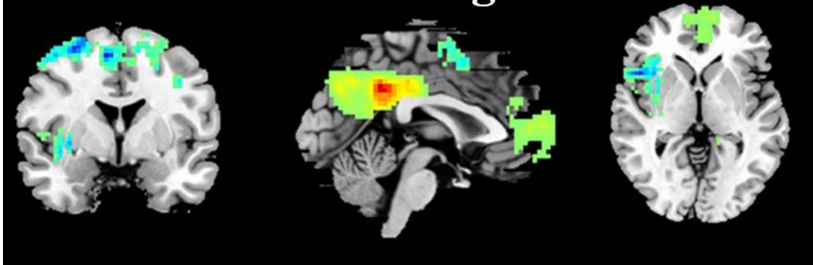

PD with MCI

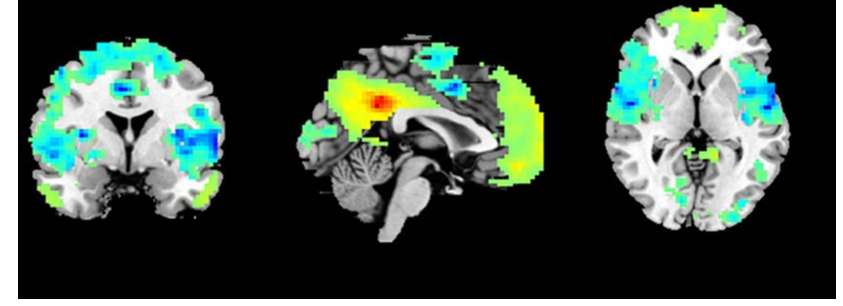

Figure I Whole-brain voxelwise pattern of the default mode network in each group. The DMN pattern for each group was obtained by using PCC (MNI coordinate: $-2,-45,34$, radius $=6 \mathrm{~mm}$ ) seed-based functional activity analysis. The random-effects one-sample $t$-test was performed and threshold was set at a corrected $\mathrm{P}<0.01$, determined by Monte Carlo simulation. 
Table 2 Descriptions of Disease-Related Differences in DMN Revealed by One-Way ANOVA

\begin{tabular}{|l|c|c|c|}
\hline Brain Region & $\begin{array}{c}\text { Peak MNI } \\
\text { Coordiates x, y, } \\
\mathbf{z}(\mathbf{m m})\end{array}$ & $\begin{array}{c}\text { Peak } \\
\text { F value }\end{array}$ & $\begin{array}{c}\text { Cluster } \\
\text { Size } \\
\left(\mathbf{m m}^{\mathbf{3}} \mathbf{)}\right.\end{array}$ \\
\hline Bilateral Precuneus & $-9,-63,51$ & 12.4 & 6615 \\
\hline
\end{tabular}

Notes: All the regions survived the Monte Carlo Stimulations, and the thresholds were set as voxel-wise $P<0.01$, cluster sizes larger than $6156 \mathrm{~mm}^{3}$.

\section{Group-Level Differences and Behavioral Significance of the Genotype-by-Disease Interaction in DMN}

Among the three groups, significant $\mathrm{FC}$ differences were detected within the BPcu $(\mathrm{F}=10.7, \mathrm{P}<0.001)$. The LSD analysis indicated that compared to PD-NC and PD-MCI, the $\mathrm{HCs}$ showed significantly increased FC within BPcu (both $\mathrm{P}<0.001$ ). Further, there was also significant difference regarding the $\mathrm{FC}$ within $\mathrm{BPcu}$ between the two $\mathrm{PD}$ groups ( $\mathrm{P}<0.001$, shown in Figure 3$)$.

As illustrated in Figure 4, for PD-MCI, the higher the FC values within BPcu were related to the better the performances information processing speed (ie, shorter time consumed of TMTA, rho $=-0.50, \mathrm{P}=0.033$, and higher scores of DSST, rho $=-0.66, \mathrm{P}=0.003$ ) and episodic memory (ie, higher scores of CFT-20 min DR, rho $=0.47, \mathrm{P}=0.047)$. Similar associations were also detected between the FC within BPcu and performances of MMSE (rho $=0.58, \mathrm{P}=0.046$ ) for PD-NC.

\section{Discussion}

The primary objective of this study was to explore the difference of pattens of anatomical and FC in HCs, PD, and PD-MCI and the relationship between the connection strengths and cognitive state. We found that in PD patients, brain network associated with the cognitive dysfunction was BPcu, and the FC within BPcu significantly decreased in PD-NC and PD-MCI. Furthermore, higher connectivity strength was associated with a faster information processing speed (TMTA, DSST), a better episodic memory (CFT-20 min DR) and a better general cognition (MMSE).

Rs-fMRI is a promising approach that measures naturally occurring low-frequency fluctuations in blood oxygenation level-dependent (BOLD) signals reflecting physiologically meaningful changes of spontaneous neural activity in the resting-state networks (RSNs) to investigate neuropsychiatric disorders. ${ }^{15}$ It avoids demanding task performance and can easily obtain relatively stable results. ${ }^{16}$ Rs-fMRI is widely used in Alzheimer's disease

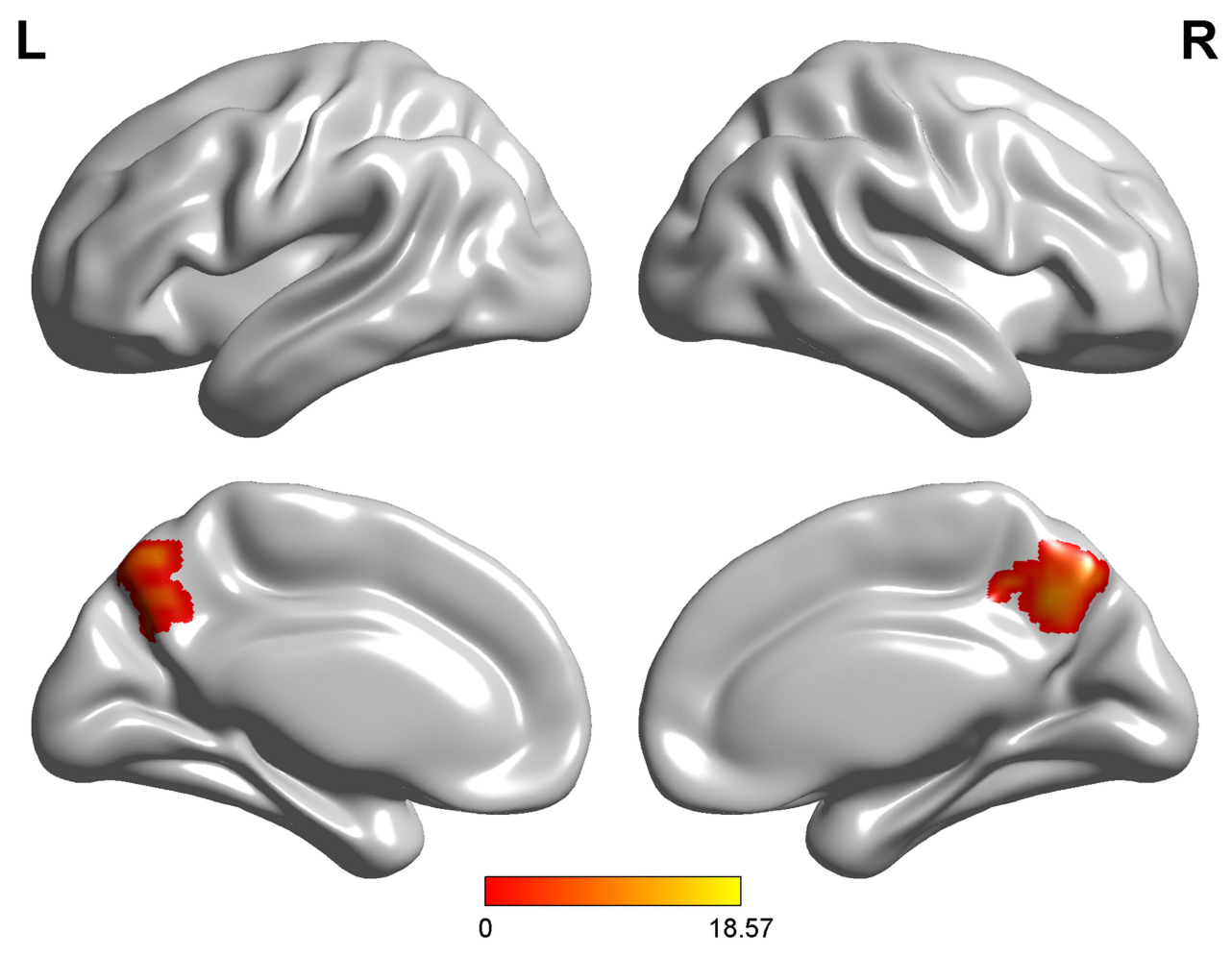

Figure 2 One-way ANOVA of DMN functional connectivity. Thresholds were set at a corrected $\mathrm{P}<0.0 \mathrm{I}$, determined by Monte Carlo simulation. 


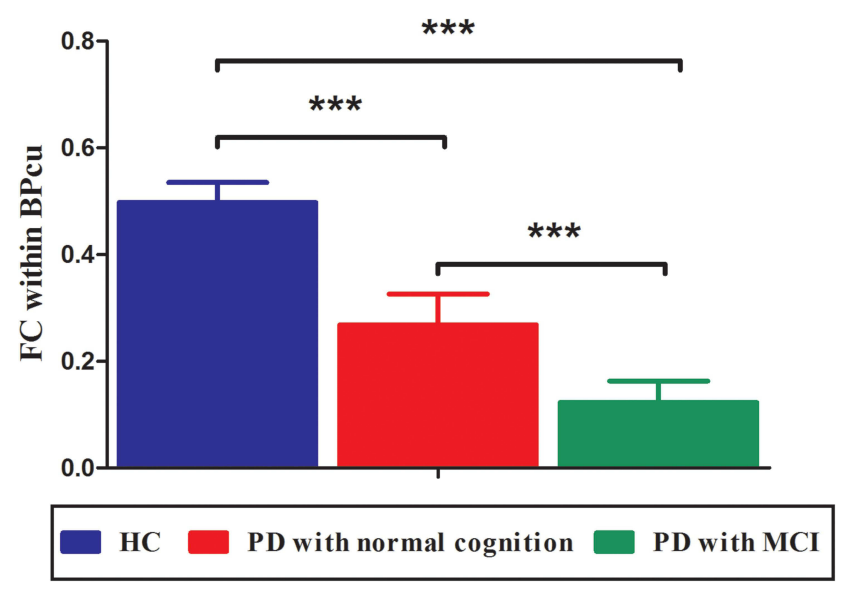

Figure 3 Group-level differences of the disease-related differences in DMN. Compared to PD patients with normal cognition and PD patients with $\mathrm{MCl}$, the $\mathrm{HCs}$ showed significantly increased FC within BPcu. Further, there was also significant difference regarding the $\mathrm{FC}$ within $\mathrm{BPcu}$ between the two PD groups. ${ }^{* * *}$ Indicate $\mathrm{P}<0.00 \mathrm{I}$.

$(\mathrm{AD}),{ }^{17}$ other dementias $^{18}$ and mood disorders. ${ }^{15}$ Mounting studies have identified a wide array of brain regions that exhibit group-wise differences between $\mathrm{HCs}$
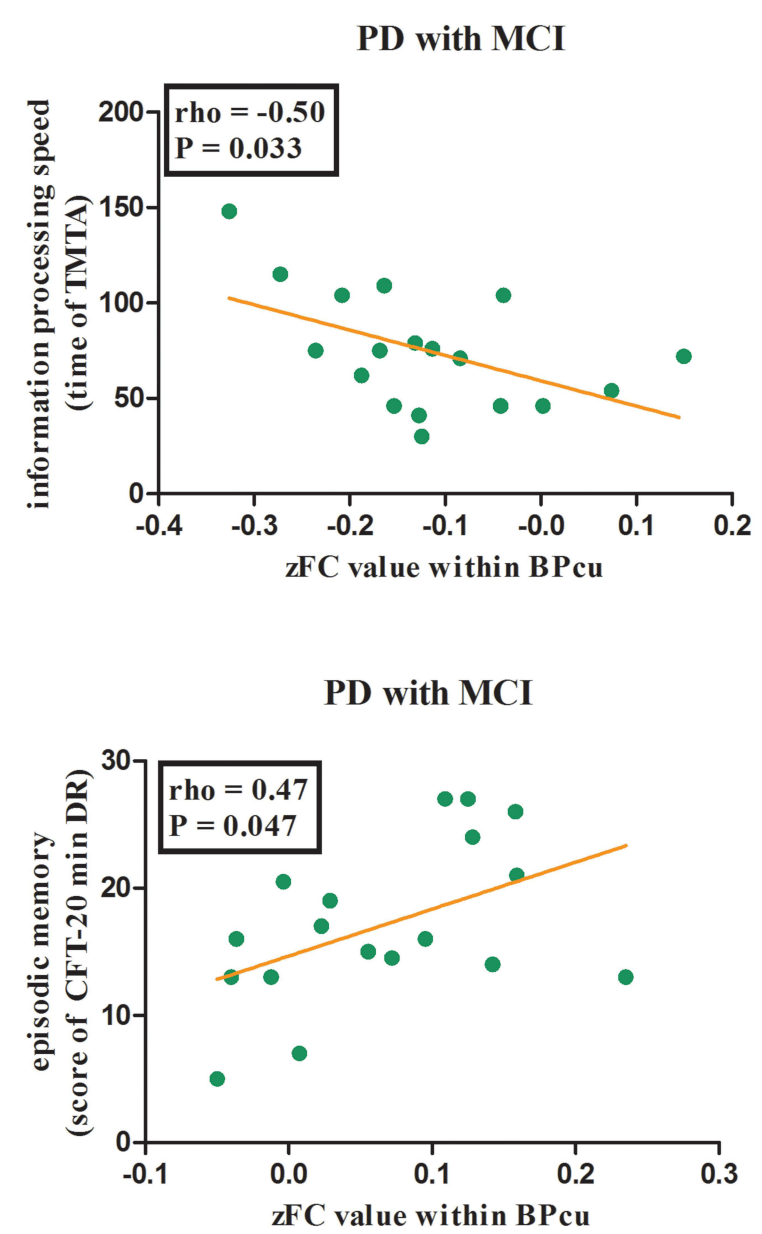

PD with MCI

PD with MCI and neuropsychiatric disorders. ${ }^{15}$ Over the last decades, rsfMRI has been carried out to investigate the FC changes in patients with PD. ${ }^{19-22}$ However, there is still a need for understanding the exact relationship between the function and brain regions and the relationship between the connection strengths and cognitive state.

$\mathrm{DMN}$ is the most popular target in RSNs, including a midline core (including the anterior medial prefrontal cortex (aMPFC) and PCC) and two subsystems (the dorsal medial prefrontal cortex (DMPFC) subsystem and the medial temporal lobe (MTL) subsystem). ${ }^{23} \mathrm{DMN}$ is thought to be involved in advanced cognitive functions. Early study has observed DMN dysfunction in PD patients. ${ }^{24}$ Wolters et $\mathrm{al}^{25}$ observed that cognitive impairment in PD was correlated with reduced FC in networks involved in cognition, especially in the DMN. Studies suggested that the precuneus was implicated in high-level cognitive functions, including self-related processing, episodic memory, and aspects of consciousness. ${ }^{26-28}$ Our
PD with MCI
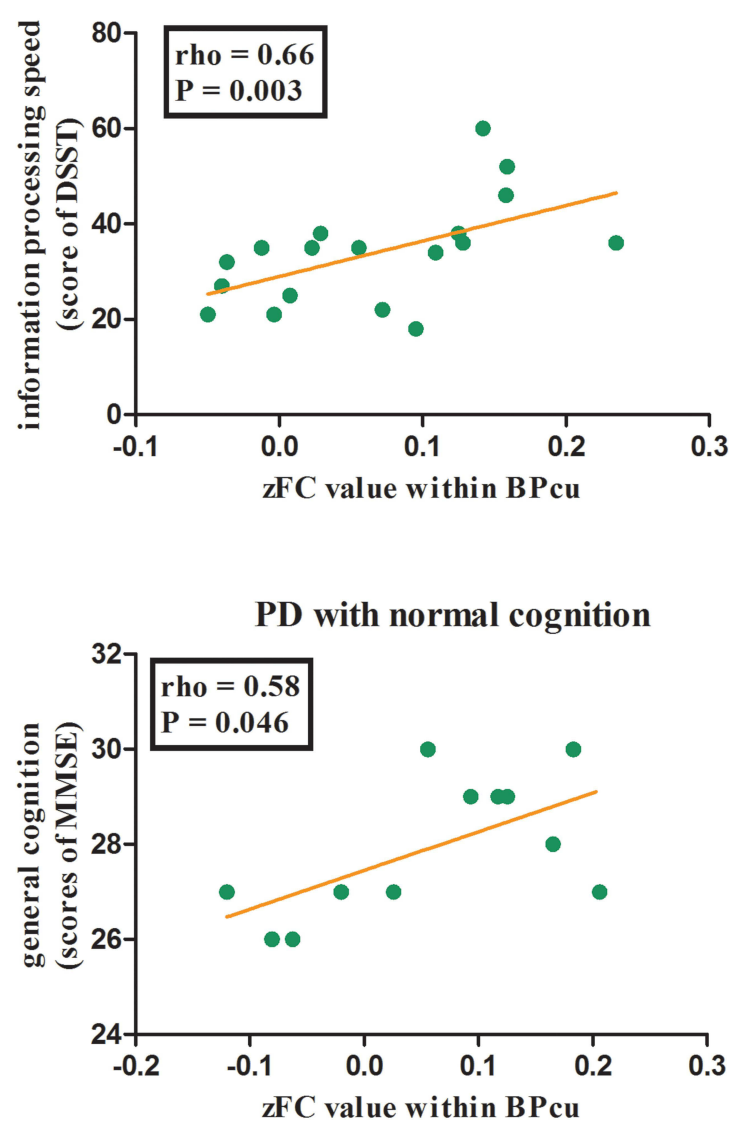

Figure 4 Behavior significances of the regions with disease-related differences in DMN. For PD patients with $\mathrm{MCl}$, the higher FC within BPcu were related to better performances of TMTA, DSST and CFT-20 min DR. For PD patients with normal cognition, the FC within BPcu were associated with scores of MMSE. 
study found that BPcu was the cognitive-related region in DMN.

There is significant clinical heterogeneity in cognitive impairment in PD29. The prevalence of PD-MCI was $25 \%$ and with the development of the disease, the cumulative prevalence of PD with dementia (PDD) gradually increases. ${ }^{18}$ The frequency and severity of PD induced cognitive decline emphasise the need to approach this impairment as a symptom that requires separate attention. However, the mechanism of PDD is not clear yet. It is urgently needed to find biomarkers or identify the risk factors or signal of PDD in early stage for improving the prognosis of the disease. ${ }^{30}$ The PD-NC and $\mathrm{HCs}$ in our study showed comparable performances of cognitive function, but the $\mathrm{FC}$ within the cognitive-related region in the brain - BPcu in PD-NC was significantly declined compared to HCs, which might be an early signal of cognitive damage. Díez-Cirarda et al ${ }^{19}$ included $26 \mathrm{HCs}, 12$ PD-NC and 23 PD-MCI and found that PD-MCI group dynamic FC deteriorated but did not been observed in PD-NC.

The cognitive impairment of PD patients is mainly manifested in visual space, attention, executive function, and memory, but these clinical manifestations are not diagnostic specific. Similar with the report, PD-MCI in our study showed significant worse performances in general cognition. For PD-MCI, the higher the FC values within BPcu were related to better performances information processing speed (ie, shorter time consumed of TMTA and higher scores of DSST) and episodic memory (ie, higher scores of CFT-20 min DR). Similar associations were also detected between the FC within BPcu and performances of MMSE for PD-NC.

There are some limitations in this study. First, this is a single center study with a limited number of patients. Second, all PD patients from this study were on medication and we cannot rule out the possibility that the drugs may have impacted the rs-fMRI. Third, MoCA scale for the global cognitive function assessment would be better while we did not collect relevant data. Four, we used 1.5T MRI, which is less sensitive than 3.0T. Lastly, the use of the outcomes needs to be further validated in additional populations.

\section{Conclusions}

In conclusion, $\mathrm{BPcu}$ was the cognitive-related region in DMN. As cognition declines, FC within BPcu weakens. For PD-MCI, the higher the FC values within BPcu were likely to be related to the better the performances of TMTA, DSST and CFT-20 min DR, which needs to be further confirmed by large-sample studies.

\section{Statement of Ethics}

The study complied with the declaration of Helsinki and was approved by the medical ethics committee of the Affiliated Jiangyin People's Hospital of Southeast University Medical College. Written consent was obtained from all participants.

\section{Funding}

This work was funded by the Guidance Plan for Social Development of Taizhou Municipal Science and Technology (ssf20160141). The funder had no role in the study design, data collection, data analysis, data interpretation, writing of the report, decision to publish, or preparation of the manuscript.

\section{Disclosure}

The authors have no conflicts of interest to declare.

\section{References}

1. Grimes D, Fitzpatrick M, Gordon J, et al. Canadian guideline for Parkinson disease. Cmaj. 2019;191:E989-e1004. doi:10.1503/ cmaj. 181504

2. Zhang ZX, Roman GC, Hong Z, et al. Parkinson's disease in China: prevalence in Beijing, Xian, and Shanghai. Lancet (London, England). 2005;365:595-597. doi:10.1016/S0140-6736(05)70801-1

3. Ma CL, Su L, Xie JJ, et al. The prevalence and incidence of Parkinson's disease in China: a systematic review and metaanalysis. J Neural Transmis. 2014;121:123-134. doi:10.1007/ s00702-013-1092-z

4. Litvan I, Bhatia KP, Burn DJ, et al. Movement disorders society scientific issues committee report: SIC task force appraisal of clinical diagnostic criteria for Parkinsonian disorders. Mov Disorders. 2003;18:467-486. doi:10.1002/mds.10459

5. Chen B. Multi-modal MRI Study of Cognitive Dysfunction in Parkinson's Disease. China Medical University; 2017.

6. Chinese Medical Association Neurology Branch Parkinson's Disease and Movement Disorders Group; Chinese Medical Doctor Association of Neurology, Parkinson's Disease and Movement Disorders Committee. Chinese diagnostic criteria for Parkinson's disease. Chin J Neurol. 2015;48(11):1015-1019.

7. Petersen RC, Smith GE, Waring SC, et al. Mild cognitive impairment: clinical characterization and outcome. Arch Neurol. 1999;56:303-308. doi:10.1001/archneur.56.3.303

8. Albert MS, DeKosky ST, Dickson D, et al. The diagnosis of mild cognitive impairment due to Alzheimer's disease: recommendations from the National Institute on Aging-Alzheimer's Association workgroups on diagnostic guidelines for Alzheimer's disease. Alzheimers Dement. 2011;7:270-279. doi:10.1016/j.jalz.2011.03.008

9. McKhann GM, Knopman DS, Chertkow H, et al. The diagnosis of dementia due to Alzheimer's disease: recommendations from the National Institute on Aging-Alzheimer's Association workgroups on diagnostic guidelines for Alzheimer's disease. Alzheimers Dement. 2011;7:263-269. doi:10.1016/j.jalz.2011.03.005

10. Buckner RL, Sepulcre J, Talukdar T, et al. Cortical hubs revealed by intrinsic functional connectivity: mapping, assessment of stability, and relation to Alzheimer's disease. $J$ Neurosci. 2009;29:1860-1873. doi:10.1523/JNEUROSCI.5062-08.2009 
11. Shu H, Shi Y, Chen G, et al. Opposite neural trajectories of apolipoprotein E 4 and 2 alleles with aging associated with different risks of Alzheimer's disease. Cerebral Cortex. 2016;26:1421-1429. doi:10.1093/cercor/bhu237

12. Bai F, Liao W, Yue C, et al. Genetics pathway-based imaging approaches in Chinese Han population with Alzheimer's disease risk. Brain Struct Funct. 2016;221:433-446. doi:10.1007/s00429014-0916-4

13. Inkster B, Nichols TE, Saemann PG, et al. Association of GSK3beta polymorphisms with brain structural changes in major depressive disorder. Arch Gen Psychiatry. 2009;66:721-728. doi:10.1001/ archgenpsychiatry.2009.70

14. Inkster B, Nichols TE, Saemann PG, et al. Pathway-based approaches to imaging genetics association studies: WNT signaling, GSK3beta substrates and major depression. NeuroImage. 2010;53:908-917. doi:10.1016/j.neuroimage.2010.02.065

15. Takamura T, Hanakawa T. Clinical utility of resting-state functional connectivity magnetic resonance imaging for mood and cognitive disorders. J Neural Transmis. 2017;124:821-839. doi:10.1007/ s00702-017-1710-2

16. Pan P, Zhang Y, Liu Y, et al. Abnormalities of regional brain function in Parkinson's disease: a meta-analysis of resting state functional magnetic resonance imaging studies. Sci Rep. 2017;7:40469. doi: $10.1038 /$ srep40469

17. Greicius MD, Srivastava G, Reiss AL, et al. Default-mode network activity distinguishes Alzheimer's disease from healthy aging: evidence from functional MRI. Proc Natl Acad Sci USA. 2004;101:4637-4642. doi:10.1073/pnas.0308627101

18. Bai F, Zhang Z, Yu H, et al. Default-mode network activity distinguishes amnestic type mild cognitive impairment from healthy aging: a combined structural and resting-state functional MRI study. Neurosci Lett. 2008;438:111-115. doi:10.1016/j.neulet.2008.04.021

19. Diez-Cirarda M, Strafella AP, Kim J, et al. Dynamic functional connectivity in Parkinson's disease patients with mild cognitive impairment and normal cognition. Neuro Image Clinic. 2018;17:847-855.

20. Prajapati R, Emerson IA. Global and regional connectivity analysis of resting-state function MRI brain images using graph theory in Parkinson's disease. Int J Neurosci. 2020.
21. Lee $\mathrm{YH}$, Bak Y, Park $\mathrm{CH}$, et al. Patterns of olfactory functional networks in Parkinson's disease dementia and Alzheimer's dementia. Neurobiol Aging. 2019.

22. Chung SJ, Kim HR, Jung JH, et al. Identifying the functional brain network of motor reserve in early Parkinson's disease. Mov Disorders. 2020.

23. Hou Y, Yuan X, Wei Q, et al. Primary disruption of the default mode network subsystems in drug-naive Parkinson's disease with mild cognitive impairments. Neuroradiology. 2020;62:685-692. doi:10.1007/s00234-020-02378-z

24. van Eimeren T, Monchi O, Ballanger B, et al. Dysfunction of the default mode network in Parkinson disease: a functional magnetic resonance imaging study. Arch Neurol. 2009;66:877-883. doi:10.1001/archneurol.2009.97

25. Wolters AF, van de Weijer SCF, Leentjens AFG, et al. Resting-state fMRI in Parkinson's disease patients with cognitive impairment: a meta-analysis. Parkinsonism Relat Disord. 2019;62:16-27. doi:10.1016/j.parkreldis.2018.12.016

26. Cavanna AE, Trimble MR. The precuneus: a review of its functional anatomy and behavioural correlates. Brain. 2006;129:564-583. doi:10.1093/brain/aw1004

27. Vogt BA, Laureys S. Posterior cingulate, precuneal and retrosplenial cortices: cytology and components of the neural network correlates of consciousness. Prog Brain Res. 2005;150:205-217.

28. Cavanna AE. The precuneus and consciousness. CNS Spectr. 2007;12:545-552. doi:10.1017/S1092852900021295

29. Margulies DS, Böttger J, Long X, et al. Resting developments: a review of fMRI post-processing methodologies for spontaneous brain activity. Magma (New York, NY). 2010;23:289-307.

30. Chinese Medical Association Neurology Branch Parkinson's Disease and Movement Disorders Group; Chinese Medical Doctor Association of Neurology, Parkinson's Disease and Movement Disorders Committee. Chinese expert consensus on research criteria for prodromal Parkinson's disease. Chin J Geriatr. 2019;38 (8):825-831.
International Journal of General Medicine

\section{Publish your work in this journal}

The International Journal of General Medicine is an international, peer-reviewed open-access journal that focuses on general and internal medicine, pathogenesis, epidemiology, diagnosis, monitoring and treatment protocols. The journal is characterized by the rapid reporting of reviews, original research and clinical studies

\section{Dovepress}

across all disease areas. The manuscript management system is completely online and includes a very quick and fair peer-review system, which is all easy to use. Visit http://www.dovepress.com/ testimonials.php to read real quotes from published authors. 